\title{
Pressure screening and fluctuations at the bottom of a granular column
}

\author{
L. Vanel, E. Clément \\ Laboratoire des Milieux Désordonnés et Hétérogènes \\ UMR 7603 - Boîte 86 \\ Université Pierre et Marie Curie, \\ 4, Place Jussieu, 75005 Paris, France
}

August 2, 2018

\begin{abstract}
We report sets of precise and reproducible measurements on the static pressure at the bottom of a granular column. We make a quantitative analysis of the pressure saturation when the column height is increased. We evidence a great sensitivity of the measurements with the global packing fraction and the eventual presence of shear bands at the boundaries. We also show the limit of the classical Janssen model and discuss these experimental results under the scope of recently proposed theoretical frameworks.

pacs: $46.10+\mathrm{z}, 05.40 .+\mathrm{j}, 83.70 . \mathrm{Fn}$
\end{abstract}

\section{Introduction and background}

Mechanical description of static granular assemblies is an old but still open problem which recently got revisited from different perspectives [1]. In a classical and well established approach, practitioners of soil mechanics developed a conceptual framework in the spirit of a plasticity theory [2, 3]. This point of view based on an incremental description of stress-strain relations is suited in practice for numerical implementation. To close the description and introduce real granular effects such as dilatancy, Mohr-Coulomb plasticity etc.. , many rheological parameters have to be introduced on a semi-phenomenological basis河. Hence, the constitutive laws usually proposed are often complex and rather difficult to handle since they can be non-linear, non-differentiable and anisotropic [5, 6]. As a consequence, the outcomes usually based on numerical calculations and applied to complicated flow histories are hard to follow and explain physically. Clearly, from a fundamental point of view (maybe not on a practical point of view!), this approach is unsatisfactory and at the moment, there is a vivid interest in establishing a deeper understanding of the passage from a description of local granular contacts and force distributions, to a macroscopic description of stress-strain relations [7], 8]. A general feature observed both in experiments [9, 10, 11, 12, 13] and in simulations [14, 15, 16, 17] is the very heterogenous and anisotropic character of the force network arising from the intergranular contact geometry and the frictional properties of these forces. The difficulty of the problem is such that no rigorous macroscopic theory of granular mechanics is so far available. Moreover, how this disorder relates to the dispersion and the fluctuations of macroscopic stress measurements is an important issue that remains open [18, 19]. Recently, based on phenomenology and properties of symmetry, theoretical works have suggested new sets of closure relations for mechanical equations which aim to render the existence of long range anisotropic structures in static assemblies, commonly referred 
to as vaults. These relations contain a large set of possible histories for the granular material[20, 21]. Besides, the passage from a microscopic to a macroscopic description was undertaken using various models of force networks, the resolution of which is based on cellular automatons [22, 23, 24], stochastic toy-models [17, 25] and analytical solutions of stochastic stress transport equations [26].

The case of a container filled with grains is a basic situation where the presence of boundaries is a natural initiator of a pressure screening effect that Janssen described last century in a pioneering contribution [27]. Quite surprisingly, the simple Janssen analysis is still used as a basis for the design of silos [28, 29] despite many difficulties in testing any models which come from a lack of reproducible experiments. Large fluctuations of the mean vertical pressure are generically observed in silos [30]. Data shows large differences for tests in identical conditions [31] and a strong dependency on the filling procedures [32]. The numerous theoretical refinements proposed to improve the Janssen analysis (see for example Ref. [4]) fail to take into account the existence of widely scattered pressure measurements and, in our opinion, this is a reason why an attempt to comparatively differentiate all theoretical predictions is most often doomed to failure [33].

In this work, we design an experimental set-up with the goal of precisely and reproducibly measuring the mean pressure as well as its fluctuations at the bottom of a granular column. We present two sets of well defined procedures which allow to obtain reproducible measurements. We quantitatively analyze these results in the form of an adaptation of the Janssen's classical model, and we discuss several theoretical models that could be used to compare our results with.

\section{Experimental set up}

\subsection{Apparatus description}

A sketch of the experimental set-up is shown on Fig.1. A mass $M$ of grains is poured into a vertical cylinder of inner radius $R=20 \mathrm{~mm}$ and lies on the horizontal top surface of a piston. The piston is designed in such a way that it never touches the cylinder walls. Thereby, the average pressure of the granular material on the piston is entirely transmitted to an electronic scale. The scale is a horizontal beam of high effective stiffness $K=2.10^{4} \mathrm{~N} / \mathrm{m}$, the deformation of which is detected with a strain gauge bridge which has the property to eliminate temperature drift effects in strain measurements. The corresponding force is measured in unit of mass with a precision $\Delta m=0.1 \mathrm{~g}$ and it will be referred to as the apparent mass $M_{a}$, since its value is different from the total mass $M$ of the granular medium. The system "beam+piston" rests on a mechanical elevator whose vertical displacement $\Delta Z$ can be varied and measured with a micrometric screw.

\section{$2.2 \quad$ Filling procedure}

We used this apparatus to measure the apparent masses of various species of grains such as monodisperse glass spheres, polydisperse iron shots or rugged quartz grains. All the results presented in this paper concern monodisperse glass spheres of diameter $d=2 \mathrm{~mm}$. In order to vary the initial packing fraction, two filling methods are used (see sketch on Fig.1). In method 1, the grains fall from a hopper located on the top of the column and due to the ramming effect of falling grains a rather compact packing is obtained. In method 2, the grains fill first an intermediate inner cylinder that initially rests on the piston. Next, the inner cylinder is slowly removed so that the grains gently flow into the outer cylinder and settle rather loosely.

\subsection{Volume fraction measurements}

Throughout the experiments, the average volume fraction of the grains was estimated by monitoring the total height $H$ of the granular column. The top level of filling is detected using a digital vernier calliper (precision $0.01 \mathrm{~mm}$ ). We checked that within a precision of one bead radius, the top surface was almost flat. This is probably a consequence of the rather small ratio between the column radius 
and the bead size : $R / d=10$ which prevents the development of a well defined slope at the free surface. A thin and flat cardboard disc of mass $m=0.3 \mathrm{~g}$ was used to obtain a well-defined top surface and reduce perturbations from the rod when coming in contact with the granular material. The bottom displacement of the column is known from the elevator's micrometric screw. The average granular density is therefore computed as $\rho=M / \pi R^{2} H$, and the packing fraction is then $\nu=\rho / \rho_{b}$, where $\rho_{b}$ is the grain density. The height measurement is always done after the corresponding apparent mass measurement since the latter is very sensitive to all kind of perturbations as we will see in the following.

\section{Obtention of reproducible pressure measurements}

\subsection{Measuring stresses in granular assemblies}

There is a fundamental and technical difficulty in measuring stresses in granular assemblies. One physical reason is rooted in the hysteretic character of the local friction forces between the grains and with the system boundaries which prevent the system from returning to the initial equilibrium after a perturbation. We stress that it is not necessary to reach plastic deformation on a large scale in order to observe an irreversible change in the equilibrium state. For instance, a simple springmass system with friction displays hysteretic properties [34 and the coupled frictional and elastic contacts between grains are expected to display similar features [35]. Since every pressure probe is by construction associated with some displacement of a reference surface like a membrane or a piston, the measuring process may modify in return the force distribution in an irreversible way and therefore, the measurement values will depend on the specific perturbation history of both the probe and the material. One remedy could be in a quest for the least disturbing probe like a very stiff spring or a feedback device where position is controlled, but this might create other problems such as pathological coupling with temperature (due to thermal dilation 36]), a decrease in the sensitivity and/or incontrollable instabilities. This is exactly the kind of problem we address in this article. We consider the granular column and the pressure probe as a paradigmatic situation where all the difficult aspects of solid friction, boundaries and pressure measurement are a priori coupled in a complex way. The basic idea of the measurement device we propose, and in particular the use of a mechanical elevator, is to produce a statistical distribution of the many metastable states corresponding to a specific average equilibrium with the piston. We want to know, for example, whether the dispersion and non-reproducibility of results currently reported in the literature is due to an intrinsically wide distribution of metastable states or if other "hidden parameters" should be considered to rationalize the results. In the following, we report two distinct ways of producing controlled and reproducible measurements, and furthermore, we describe basic observations that could explain why previous experiments have yielded non-reproducible data sets.

\subsection{Measurement procedures}

Two different kinds of experimental procedures are investigated. Both lead to a set of reproducible results within a rather small fluctuation scale. They are both based on a slow downward displacement of the system "beam+piston" at a slow velocity (typically $20 \mu \mathrm{m} / \mathrm{s}$ ). Note that importantly, the granular column seems to slide as a whole since we realize that any downward displacement of the bottom piston is also evidenced as a simultaneous downward displacement of the column's top. This procedure has a-priori two remarkable advantages: (i) the kinetic energy of the falling grains during the filling stage which is partially stored as elastic energy of the beam, is relaxed, and therefore there is a memory loss of the initial pouring dynamics; (ii) the downward motion of the piston allows the granular column to slide down such that the friction forces at the walls should be fully mobilized and directed upwards. This is crucial for a quantitative analysis since generally, the theoretical models assume that the granular material is at the sliding limit at the walls. This important step was 
missing in previous experimental reports that we know. Generally speaking, the system would reach an equilibrium position depending specifically on the kinetic energy due to the pouring procedure, the effective distribution of friction forces at the boundaries, and the actual displacement of the pressure probe (related to the spring stiffness).

Note that in a preliminary set of experiments [36], we reported a large coupling of the pressure with small temperature variations. We linked this effect to problems of differential thermal dilation between different parts of the setup. Therefore, a double isothermal container was used to reduce the drastic effect of temperature on the plexiglass column as much as possible (this material has a large dilation coefficient and is a poor temperature conductor). In the new set-up, problems of temperature are dampened using a material with a very low temperature dilation coefficient such as an iron-nickel alloy. It turns out that this precaution is sufficient to obtain measurements independent of the actual temperature values. In fact we observe that after each downward displacement, there is a time gap of typically $30 \mathrm{~s}$ which is large enough to relax our mechanical system and to make a measurement, but which is small enough to be able to neglect the effects of temperature drifts.

\subsubsection{Descent experiments}

The first kind of experiment probes the effect of accumulated series of downward displacements of the piston. It will be referred to as the descent experiment. Note that in a preliminary report, the procedure has been presented as well as some of the effects we discuss in the following [37]. The vertical displacements are performed with a fixed amplitude of $0.125 \mathrm{~mm}(1 / 16$ of bead size) on a total distance of $20 \mathrm{~mm}$ corresponding to one column radius. The displacement amplitude has been chosen for practical convenience, but we checked that changing its magnitude does not modify the reported behavior. Fig. 2a shows a typical evolution of the apparent mass both during the displacement of the piston and after the piston was stopped. We observe that when the piston is slowly moved down, the apparent mass abruptly decreases. The accelerated motion communicated to the system piston+scale during this process is responsible for a slight decompression of the scale spring which leads to this abrupt decrease. As soon as the elevator stops, the apparent mass suddenly increases up to a point where a slow relaxation of the apparent mass occurs and a stable value is finally reached. Since a decrease in the apparent mass implies an upward motion of the scale spring, it could be concluded that after the slow relaxation the friction forces are not fully mobilized at the walls. Nevertheless, as a practical statement, we note that the amplitude of the relaxation effect on the apparent mass values is less than $2 \%$ so that the friction would be in any cases almost fully mobilized. Moreover, a decrease of the apparent mass means that the overall screening effect becomes stronger. This is in contradiction with a loss of friction mobilization and leads us to think that the small upward displacement of the piston is not really disturbing. One should also keep in mind that this displacement is less than $0.5 \mu \mathrm{m}$, which is smaller than the typical distance to mobilize friction [38]. In fact, the increase of the screening effect during this slow relaxation may correspond to a slow reorganization of the packing structure, but this point deserves a more thorough study. In this report, we only concentrate on the final values of the apparent mass as shown on Fig. 2a. Fig. 3 shows typical plots of the apparent mass as a function of the piston displacement for a filling mass $M=300 \mathrm{~g}$. This filling corresponds to a height : $H \simeq 8 R$. Whatever filling method is used, there is a global increase of the apparent mass with the number of displacement steps and, eventually, it seems a steady state is reached. Around this global behavior, fluctuations of a smaller scale are evidenced. The average packing fraction changes with the number of displacement steps and also tends towards a steady state. When the initial packing is rather compact $(\nu \simeq 0.63)$, a global decompaction occurs, while there is a global compaction when the initial packing is rather loose $(\nu \simeq 0.58)$. Furthermore, whatever the initial packing fraction, the final packing fractions tend to be the same. The reproducibility of the method can be evidenced on Fig. 3a where the results of two independent experiments are superposed. 


\subsubsection{Tapping experiments}

The second kind of experiment probes the effect of changing the granular density with the use of vibrations produced by series of taps on the container walls. It will be referred to as the tapping experiments. Starting from a loose piling, previous studies have shown that series of taps induce a slow compaction effect[39]. Note that in this last reference, tapping was a vertical impulse; here, we use series of side impacts. The typical evolution of the apparent mass is shown on Fig. 2b. After a tap, there is a sudden increase of the apparent mass. The main reason for this increase is due to an hysteresis effect of the measurement device. A tap on the wall can either break the frictional contacts between the grains and the walls or make the piston vibrate. In both cases this should result in an additional downward compression of the piston. After a tap, the piston cannot decompress and return to its initial equilibrium because of the granular column frictional resistance to motion. In order to release the dynamical compression effect due to a tap, the piston is then slowly moved down and as a consequence, there is a fast decrease of the load to a level which is quite reproducible. A remarkable fact is that the final apparent mass (after a small downward displacement) is independent of the impact itself but is very well correlated to the average volume fraction, and this for the many independent experiments that we did. On Fig. 2b, we also display the mass variation obtained from the initial pouring procedure. The corresponding data points are seen on this figure for a time less than $50 \mathrm{~s}$. We observe that the apparent mass indeed saturates at a value of about $80 \mathrm{~g}$. Nevertheless, we tested that this value is difficult to reproduce from one filling to the other. On the other hand, after the first small and slow displacement $\Delta z$, we obtain a value $50 \%$ smaller which is reproducible from one experiment to the other within a $5 \%$ error bar! On Fig. 4, we display a typical plot of the apparent mass $M_{a}$ as a function of the packing fraction for a filling mass $M=300 g$. Two independent sets of data are shown to illustrate the reproducibility of the variation as well as the level of fluctuations. The tapping procedure leads to a progressive compaction of the granular column while the apparent mass is decreasing.

\section{Analysis of the Experimental results}

\subsection{A phenomenological description and a qualitative interpretation}

The results obtained in both descent and tapping experiments can be summarized on a unique diagram. Fig. 5a shows such a diagram where the apparent mass is plotted as a function of the packing fraction for a filling mass $M=300 \mathrm{~g}$. The arrows indicate the direction of evolution in the course of each experiment. A similar qualitative diagram is obtained with many other granular materials like polydisperse steel spheres or angular quartz grains, but we only report the results for $d=2 \mathrm{~mm}$ glass spheres here. There is clearly a drastic change in the static equilibrium with the experimental procedure followed. Data for the tapping experiments shows that a density increase of about $8 \%$ induces a pressure decrease of about $20 \%$ (Curve $\{1\}$ ). On the other hand, the descent experiments show that density variations of less than $5 \%$ induce a pressure increase as large as $50 \%$ (Curves $\{2\}$ and $\{3\}$ ). Moreover, this last procedure shows a compaction effect for initially loose packings and a decompaction effect for initially dense packing. Experiments on a 2D Schneebeli medium show than in a descent experiment like ours, friction at the wall produces shearing bands [40]. In these experiments, the assembly was initially rather compact and due to shearing, a global decompaction occurred creating a radial density profile with a looser density on the edges and a larger density at the center. We suppose that in our descent experiments, a similar shearing effect occurs. Moreover, our results are consistent with the classical vision of soil mechanics where many standard tests have shown that shearing a dense medium produces a decompaction[41] and shearing a loose medium produces a compaction[2]; this is what happens in curves $\{2\}$ and $\{3\}$. In the limit of large deformation a so-called "critical density", independent of the initial stage, is reached. This is probably what could happen around $\nu o \simeq 0.593$ where both curves cross. In our case, it simply 
indicates that an identical density profile might be finally reached from both sides. On the other hand, what happens in $\{1\}$ is a priori less clear. The way we understand these results is that the vibrations produce a compaction effect which "kills" the density gradients developed through the shear bands. After a tap, the displacement of the piston is small enough to avoid the formation of shear bands, and even if a shear band was initiated, the next vibrational shock is likely to destroy it. As a consequence, we have a more homogeneous granular packing along the radius and likely in the whole column. This interpretation is consistent with the fact that the end of curve $\{1\}$, corresponding to a sequence of tapping and descents, seems to join the beginning of curve $\{3\}$ which represents an early situation when the shear bands are not yet developed. In the tapping experiment, we observe a decrease of the apparent mass when the density is increased, which witnesses a stronger screening effect of the boundaries. Such a dependence between friction and density is reminiscent of recent experimental results [42] in which the extraction force of a rod buried in sand is shown to increase drastically with the packing fraction. At this point, to go beyond these many conjectures, more information on the local density spatial distribution would be needed. We are in the process of measuring this experimentally using an invasive technique.

\subsection{Quantitative study of the pressure saturation}

\subsubsection{Comparison with the classical Janssen model}

In the following, we study the apparent mass as a function of the total mass poured in the cylinder. As a guide-line for describing the results, we present at first the Janssen classical analysis[27]. Last century, Janssen proposed a simple heuristic argument to account for pressure distribution $P(z)$ at the edges of a container filled with a granular material. The argument is based on mechanical equilibrium of a horizontal slice of thickness $d z$ and horizontal surface area $S=\pi R^{2}$. The slice is submitted to the action of its own weight, a pressure gradient from the surrounding granular material and friction forces $d F_{\text {frict }}$ from the lateral walls such that:

$$
-\nabla_{z} P(z) . S d z+\rho g S d z-d F_{\text {frict }}=0
$$

where $\rho$ is the granular density. The core of the model assumes that the vertical pressure is transferred into a horizontal pressure via a constitutive Janssen coefficient $K$ and that the friction forces are fully mobilized in the upward direction. Thus the friction force acting on a surface element $d S=2 \pi R d z$ is: $d F_{\text {frict }}=\mu K P(z) 2 \pi R d z$, where $\mu$ is the solid friction coefficient of the grains with the wall. A rough measurement of the coefficient of friction between glass beads and the wall gives $\mu=0.4( \pm 0.02)$. Integration of equation(1) with the boundary condition $P(0)=0$ yields an exponential saturation of the pressure with depth $z: P(z)=\rho g \lambda(1-\exp (-z / \lambda))$. A central parameter in the theory is the characteristic length $\lambda$ which accounts for a pressure screening effect due to the boundaries: $\lambda=R / 2 \mu K$. In fact, the theory assumes that the stress distribution is uniform across any horizontal section of the material. This assumption turns out to be false and many theoretical refinements have aimed to correct it (see, for example, ref. [4]). However, the predicted pressure saturation curves are qualitatively very similar to each other. In the following, we only consider the simple saturation equation predicted by Janssen, except that it is expressed in unit of mass. The saturation mass, $M_{\infty}=\rho \pi R^{2} \lambda$, the mass $M$ of grains poured inside the column and the measured apparent mass $M_{a}$ should agree with the equation :

$$
M_{a}=M_{\infty}\left(1-\exp \left(-M / M_{\infty}\right)\right)
$$

We experimentally tested this law by varying the mass of grains in the silo. To change the filling mass, the silo was entirely emptied and filled again with the desired amount of grains. Hence, data obtained for different filling mass corresponds to completely independent sets of experiments. Since 
we have good reasons to believe that the packings obtained are more homogeneous, we now focus on the data obtained with the second measurement procedure, i.e. the tapping experiments. On Fig. 6, the apparent mass is plotted as a function of the filling mass for an average packing fraction $\nu=0.585 \pm 0.005$. Note that the straight line on the picture would represent a perfectly hydrostatic behavior. We clearly observe a saturation of the bottom pressure as the most elementary Janssen vision would predict. On the other hand, at a quantitative level, we evidence that the experimental data points are systematically above Janssen equation (2) when the fitting parameter $M_{\infty}$ takes the value of the experimental saturation mass (see dashed curve on Fig.6 obtained with: $M_{\infty}=53 \mathrm{~g}$ ). This remains true for every saturation curve we obtained with other volume fractions.

\subsubsection{Analysis using a modified Janssen model}

To account for the experimental fact that the hydrostatic behavior is more pronounced than Janssen prediction, we propose a two-parameter model where is explicitly introduced a finite-size zone the apparent behavior of which is purely hydrostatic. At the most elementary level, the hydrostatic zone is viewed as an horizontal slice of mass $M_{0}$ located at the bottom of the column, while the rest of the column is supposed to behave according to Janssen's differential equation (11). The resulting equations are :

$$
\begin{aligned}
& \text {-for } M \leq M_{0}, M_{a}=M, \\
& \text {-for } M>M_{0}, M_{a}=M_{0}+M_{\infty}^{C}\left[1-\exp \left(-\left(M-M_{0}\right) / M_{\infty}^{C}\right)\right]
\end{aligned}
$$

The thin solid curve on Fig. 6 is a best fit of the experimental data points with the previous equation (3). The corresponding values of the fitting parameters are $M_{0}=13.4 \mathrm{~g}$ et $M_{\infty}^{C}=39.6 \mathrm{~g}$. Although the two-parameter model is based on a very crude assumption, the fit is quite good and clearly captures the main features of the experimental results. From the data obtained with the many tapping experiments we performed, we measured the corresponding sets of parameters: $M_{0}$ and $M_{\infty}^{C}$, for other values of the packing fraction. The dependency of the fitting parameters with the packing fraction is shown on Fig. 7 . We find that $M_{0}$ has only a slight tendency to decrease with $\nu$ while the parameter $M_{\infty}^{C}$ is clearly a decreasing function of the packing fraction. From the values of $M_{\infty}^{C}$ and the relationship $M_{\infty}^{C}=\rho \pi R^{3} / 2 \mu K_{\text {eff }}^{C}$, an effective Janssen coefficient $K_{\text {eff }}^{C}$ is determined with the hypothesis that the coefficient of friction is independent of the packing fraction. As a comparison, we also determined the effective Janssen coefficient $K_{\text {eff }}$ obtained with the original Janssen model where the saturation mass $M_{\infty}$ is simply the sum of $M_{\infty}^{C}$ and $M_{0}$. In both cases, the effective Janssen coefficients increase with $\nu$ (see Fig. 8).

\section{Discussion}

In the previous report we experimentally determined the shapes of the saturation curves for columns in static equilibrium with packings of different densities. We evidenced that the effective form of the saturation curve is strongly dependent on the density and as a rough statement, we find (i) that the effective screening length has a tendency to decrease when the density increases, and (ii) that the saturation curves stay very close to the hydrostatic curve for small fillings. We are aware that the use of a two-parameter model to fit the data is not really satisfactory. Nevertheless we believe that this investigation can be useful since any interpretative theoretical model should be consistent with these experimental facts and moreover, any theoretical saturation curve analyzed with our two-parameter model should provide relations identical to the one obtained on Fig. 8. However, from a classical perspective, we found a priori surprising that $K_{\text {eff }}$ stays smaller than unity while $K_{\text {eff }}^{C}$ is almost always larger than unity. In the engineering literature 㘬, it would be said that the granular material is in an active state when $K<1$ and in a passive state when $K>1$. The real physical meaning of 
this classification is usually related to the mobilization of friction forces inside the granular material and, a priori, it is not obvious to which case our experiments should correspond to.

Recently a theoretical prediction was made on the existence of a pronounced hydrostatic zone for a column filled with grains of finite elasticity [43]. According to this theory, there is a zone at the top and at the bottom of the column where the grain displacements relative to the walls are much smaller than the minimum distance necessary to mobilize friction. Here we are under the impression that our descent procedure should prevent such a condition to occur. Another physical explanation for the hydrostatic zone could be the following. Let us assume that the stresses actually propagate along specific paths. The qualitative argument is that the weight of grains close to the piston can be carried along stress paths that will never reach the walls but instead, directly hit the piston. Then some of the mass cannot be screened by the walls so that the real saturation curve should be closer to the hydrostatic curve as compared to a simple Janssen law. Several models actually predicts that the stresses propagate along specific directions. This is the case of a model Bouchaud et al [20] have proposed recently, but it is also a consequence of the classical I. F. E. hypothesis (Incipient Failure Everywhere). In addition, Socolar has found using a stochastic toy-model that the average vertical force in a silo is closer to the hydrostatic curve than the Janssen prediction. The curve predicted by Socolar in Fig. 4 of ref. 25] and our experimental curve in Fig. 6 are very similar. Socolar also observed the emergence of long stress chains which are reminiscent of experimental observations [9]. Those stress chains represent stress paths along which large values of stress are carried. Thus, the qualitative interpretation we described above seem to be supported by Socolar's results. The question arises whether other theories which don't predict the existence of stress chains can also describe the pronounced hydrostatic behavior we observed experimentally. A precise quantitative comparison between various theoretical models and our experimental data is the aim of a subsequent paper 44].

\section{Conclusion}

In this paper we investigate the fluctuations and variations of the average pressure at the bottom of a granular column. First we design an apparatus and two experimental procedures which allow to obtain reproducible measurements. For both procedures, we are able to separate a level of fluctuations and a level of systematic variation which are very well correlated with the average packing fraction. One of these operating modes is thought to provide information on rather homogeneous packings and we perform a quantitative analysis of the pressure saturation curves for different packing fractions. We find that the data points are systematically above the simplest prediction one can make using a Janssen model. We make a simple extension of this model and introduce an effective hydrostatic zone at the bottom of the column as a new fitting parameter. The agreement with the experimental results is quite good and we find that the Janssen constant which is the second fitting parameter, shows a systematic increase with the packing fraction. On the other hand, the bottom hydrostatic mass shows a weak variation within the experimental errors.

The second experimental procedure is thought to achieve a situation where the downward motion of the grains and the friction with the boundaries produce localized shear bands. Therefore a systematic variation of the density is expected along the column radius, i.e. the packing fraction at the walls is less than at the center. Although this density variation is very small, the effect on the mechanical equilibrium is drastic since the apparent saturation mass shows a systematic increase and reaches values which are twice as large as the one obtained with an homogeneous column. We are aware that this series of experiments and the interpretation we propose, though encouraging, are preliminary. The fact that we find reproducible data sets calls for more experimental work with other materials, other boundary conditions and larger column sizes. Moreover, we need a systematic series of tests in order to discriminate clearly between all different theoretical approaches and possibly reach a well established vision of this elementary but still unraveled problem of the static equilibrium of a 
granular assembly in a column 44].

\section{References}

[1] For recent contributions and discussions on this issue see: Physics of Dry Granular Media, ed. by H. J. Herrmann, J.-P. Hovi and S. Luding, Kluwer Acad. Publisher (1998).

[2] A. Schofield, and P. Wroth, Critical State Soil mechanics, (McGraw Hill, 1968).

[3] J. Feda, Mechanics of Particular Materials : The Principles, Elsevier (1982).

[4] R. M. Nedderman, Statics and Kinematics of Granular Materials, Cambridge University Press (1992).

[5] D. Harris, J. Mech. Phys. Solids 40 (2), 273 (1992).

[6] A. N. Norris, and D. L. Johnson, J. Appl. Mech. 64, 39 (1997).

[7] P. A. Cundall and O. D. L. Strack, in Mechanics of Granular Materials : New Models and Constitutive relations, p113, ed. by M. Satake and J. Jenkins, Elsevier (Amsterdam, 1983).

[8] J. Goddard, Continuum Modeling of Granular Assemblies, in ref. [1], p1.

[9] P. Dantu, Proceedings of 4th Int.Conf. Soil Mechanics and Foundation Engineering, (Butterworth Scientific Publications, London, 1957) .

[10] T. Travers, D. Bideau, A. Gervois, and J. C. Messager, J. Phys. A 19, L1033 (1986).

[11] O. Tsoungui, D. Vallet, and J. C. Charmet, in ref. [1], p149.

[12] F. Delyon, D. Dufresne, and Y.-E. Lévy, Ann. Ponts Chauss. 53-54, 22 (1990) (in french).

[13] C. H. Liu et al., Science 269, 513 (1995). D. M. Mueth, H. M. Jaeger, and S. R. Nagel, Phys. Rev. E 57, 3164 (1998).

[14] F. Radjai, M. Jean, J. J. Moreau, and S. Roux, Phys. Rev. Lett. 77, 274 (1996) .

[15] S. Ouaguenouni, and J. N. Roux, Europhys. Lett. 39, 117 (1997).

[16] F. Radjai, D. E. Wolf, M. Jean, and J.-J. Moreau, Phys. Rev. Lett. 80, 61 (1998).

[17] C. Eloy, and E. Clément, J. de Phys. I (France) 7, 1541 (1997).

[18] B. Miller, C. O’Hern, and R. P. Behringer, Phys. Rev. Lett. 77, 3110 (1996); C. T. Veje et al., in ref. [1], p 237.

[19] A.Ngadi, and J.Rajchenbach, Phys. Rev. Lett. 80, 273 (1998).

[20] J. P. Bouchaud, M. E. Cates, and P. Claudin, J. Phys. I (France) 5, 639 (1995); J. Wittmer, P. Claudin, M. Cates, and J. P. Bouchaud, Nature 382, 336 (1996).

[21] S. F. Edwards, and C.C. Mounfield, Physica A 226, 1 (1996).

[22] S. N. Coppersmith, C. H. Liu, S. Majumdar, O. Narayan, and T. A.Witten, Phys. Rev. E 53, 4673 (1996).

[23] P. Claudin, and J. P. Bouchaud, Phys. Rev. Lett. 78, 231 (1997).

[24] J. Hemmingsson, H. J. Herrmann, and S. Roux, J. Phys. I 22, 291 (1997). 
[25] J. E. S. Socolar, Phys. Rev. E 57, 3204-3215 (1998).

[26] P. Claudin, J.-P. Bouchaud, M. E. Cates, and J. Wittmer, Phys. Rev. E 57, 4441 (1998).

[27] H. A. Janssen, Z. Ver. Dtsch. Ing. 39, 1045 (1895).

[28] C. V. Schwab, I. J. Ross, G. M. White, and D. G. Colliver, Trans. ASAE 37, 1613 (1994).

[29] S. A. Thompson, N. Galili, and R. A. Williams, Trans. ASAE 39, 1093 (1996).

[30] R. L. Brown, and J. C. Richard, Principles of Powder Mechanics (Pergamon, New York, 1970).

[31] J. H. Shaxby, and J. C. Evans, Trans. Faraday Soc. 19, 60 (1923).

[32] T. Jotaki, and R. Moriyama, J. Soc. Powder Technology Japan 14, 609 (1977).

[33] D. Lenczner, Mag. Concrete Res. 15 (44), 101 (1963).

[34] J. Duran, E. Kolb, and L. Vanel, Phys. Rev. E 58, 805 (1998).

[35] R. D. Mindlin, and H. Deresiewicz, J. Appl. Mech. 20, 327 (1953).

[36] E. Clément, Y. Séréro, J. Rajchenbach, and J. Duran, in Powder and Grains 1997, Proceedings

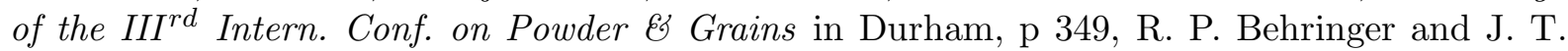
Jenkins editors, (Balkema, Rotterdam, 1997).

[37] L. Vanel, E. Clément, J. Lanuza, and J. Duran, see ref. [1], p249.

[38] P. Berthoud, and T. Baumberger, Proc. R. Soc. Lond. A 454, 1615 (1998).

[39] J. B. Knight et al., Phys. Rev. E 51, 3957 (1995).

[40] O. Pouliquen, and R. Gutfraind, Phys. Rev. E 53, 552 (1996).

[41] O. Reynolds, Phil. Mag. 20, 469 (1885).

[42] V. K. Horwarth, I. M. Janosi, and P. J. Vella, Phys. Rev. E 54, 2005 (1996).

[43] P. Evesque, and P. G. deGennes, C. R. Acad. Sci. (Paris) IIb 326, 761 (1998).

[44] L. Vanel, P. Claudin, J.-Ph. Bouchaud, M. E. Cates, E. Clément, and J. P. Wittmer, "Stress propagation in a granular column: theory and experiment", preprint cond-mat/9904094. 


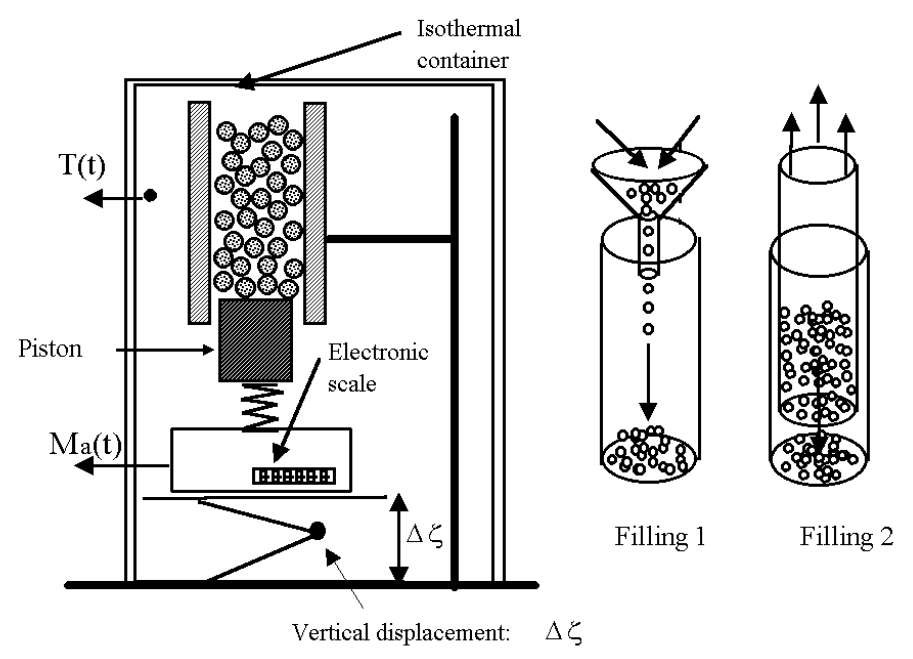

Figure 1: Left picture: sketch of the experimental apparatus. Right picture: sketch of the two filling methods where the arrows indicate the motion of the grains and/or the motion of the intermediate inner cylinder. 

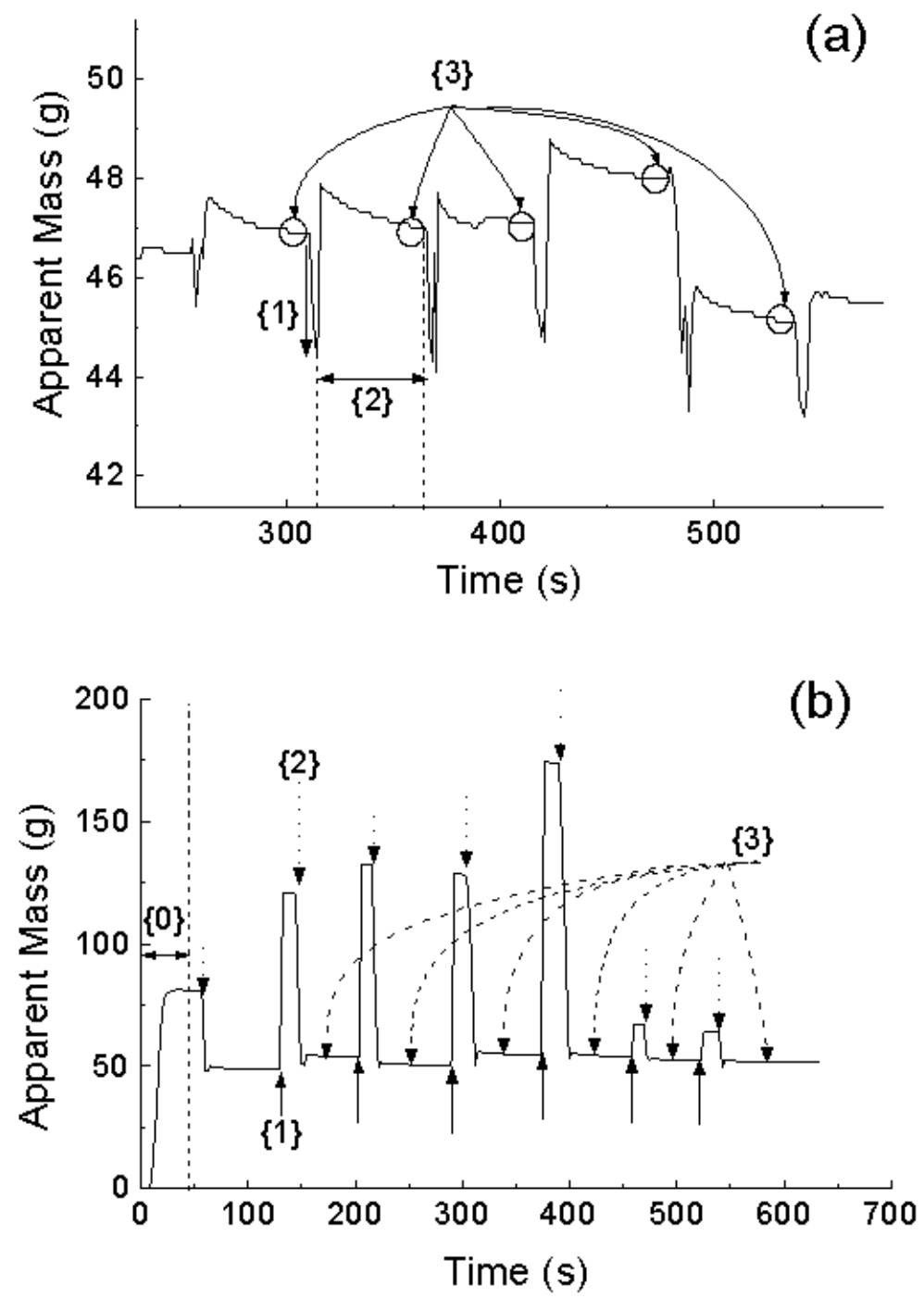

Figure 2: Evolution of the apparent mass $M_{a}$ with time: (a) during a descent experiment: $\{1\}$ indicates a descent of the piston; $\{2\}$ indicates a period of rest for the piston; $\{3\}$ indicates the measured values of the apparent mass. (b) during a tapping experiment: $\{1\}$ indicates a tap; $\{2\}$ indicates a descent of the piston; $\{3]$ indicates the measured values of the apparent mass. The $\{0\}$ sign indicate the filling stage. The discrete steps observed correspond to the finite resolution of the measuring scale. 

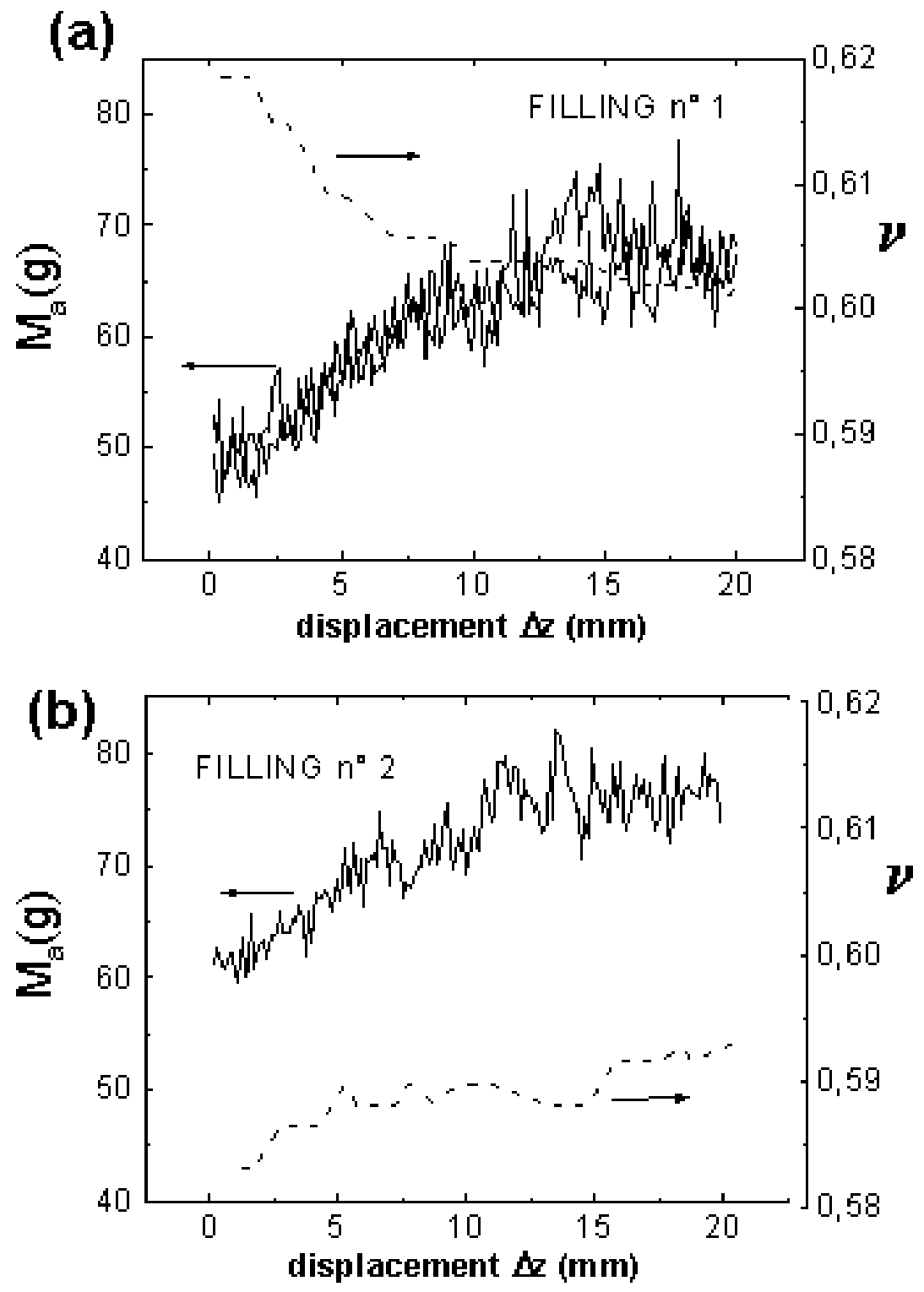

Figure 3: Descent experiment: Apparent mass $M_{a}$ (solid line) and packing fraction $\nu$ (dashed line) as a function of the vertical downward displacement $\Delta Z$ for a filling mass $M=300 \mathrm{~g}$. (a) filling method 1, the thin and the thick solid lines are two independent experiments; (b) filling method 2. 


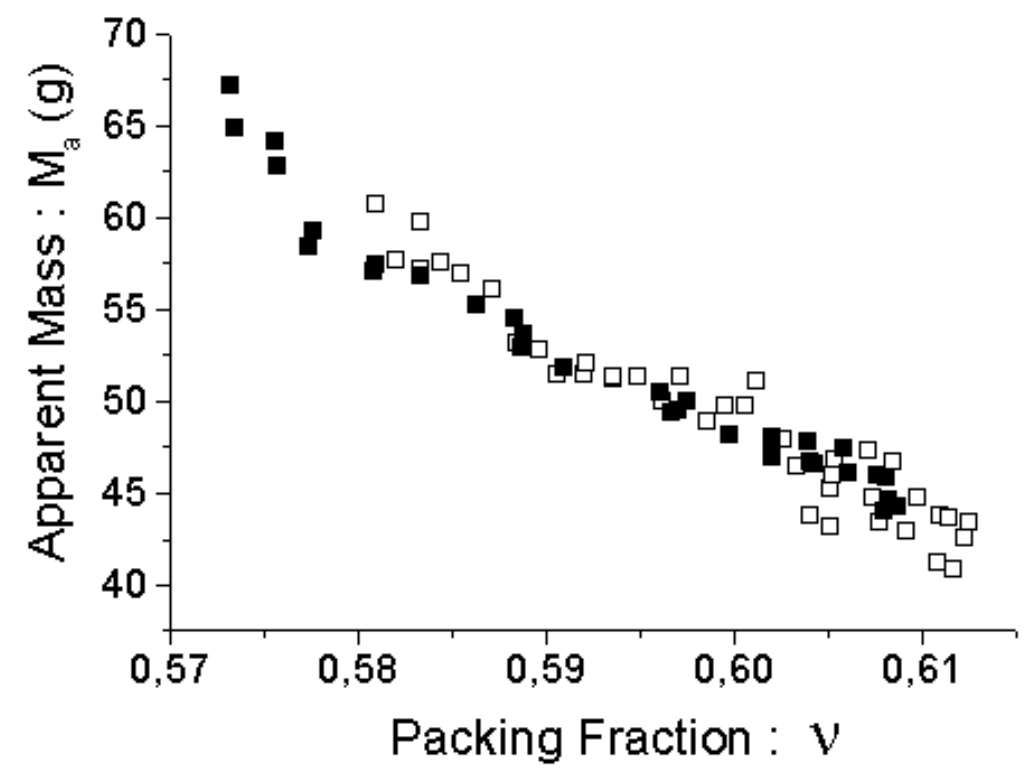

Figure 4: Tapping experiment: Apparent mass $M_{a}$ as a function of the packing fraction $\nu$ for a filling mass $M=300 \mathrm{~g}$. Open and closed squares refer to two distinct experiments.

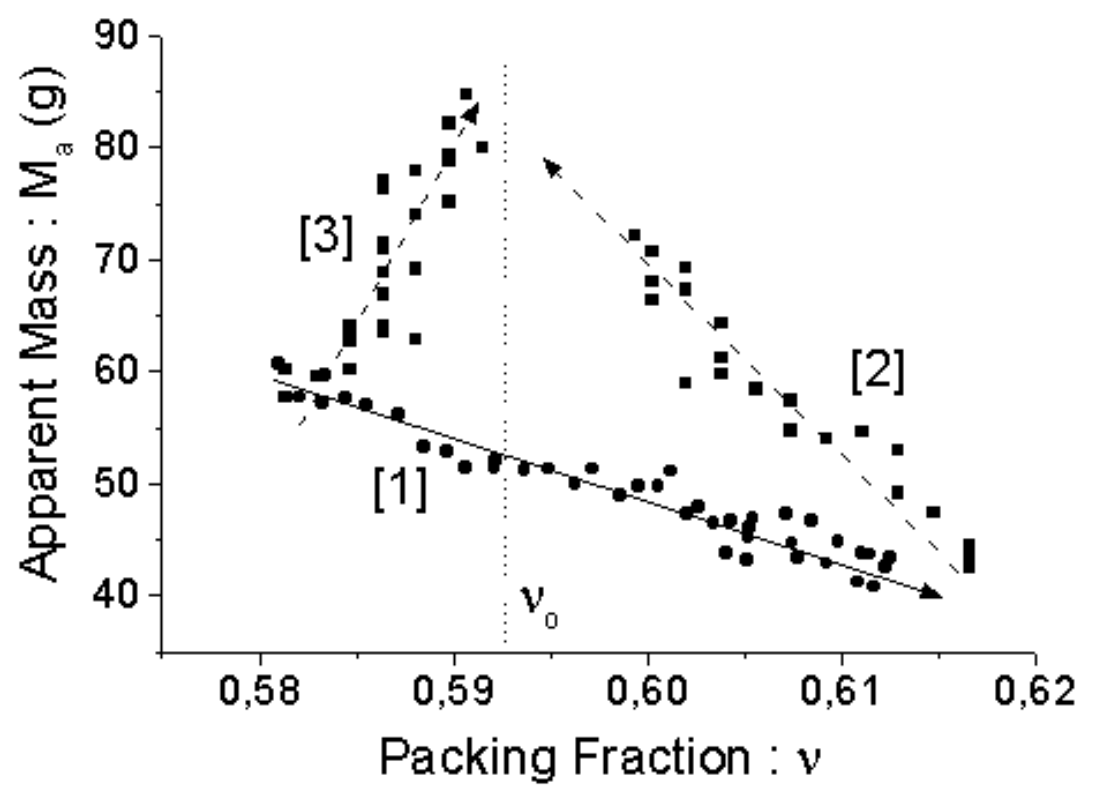

Figure 5: Apparent mass-Packing fraction diagram with a filling mass $M=300 \mathrm{~g}$ for monodisperse glass spheres. Sign [1] indicates a tapping experiment, sign [2] a descent experiment with filling method $n^{\circ} 1$, sign [3] a descent experiment with filling method $n^{\circ} 2$. Arrows indicate the evolution in the course of each experiment. 


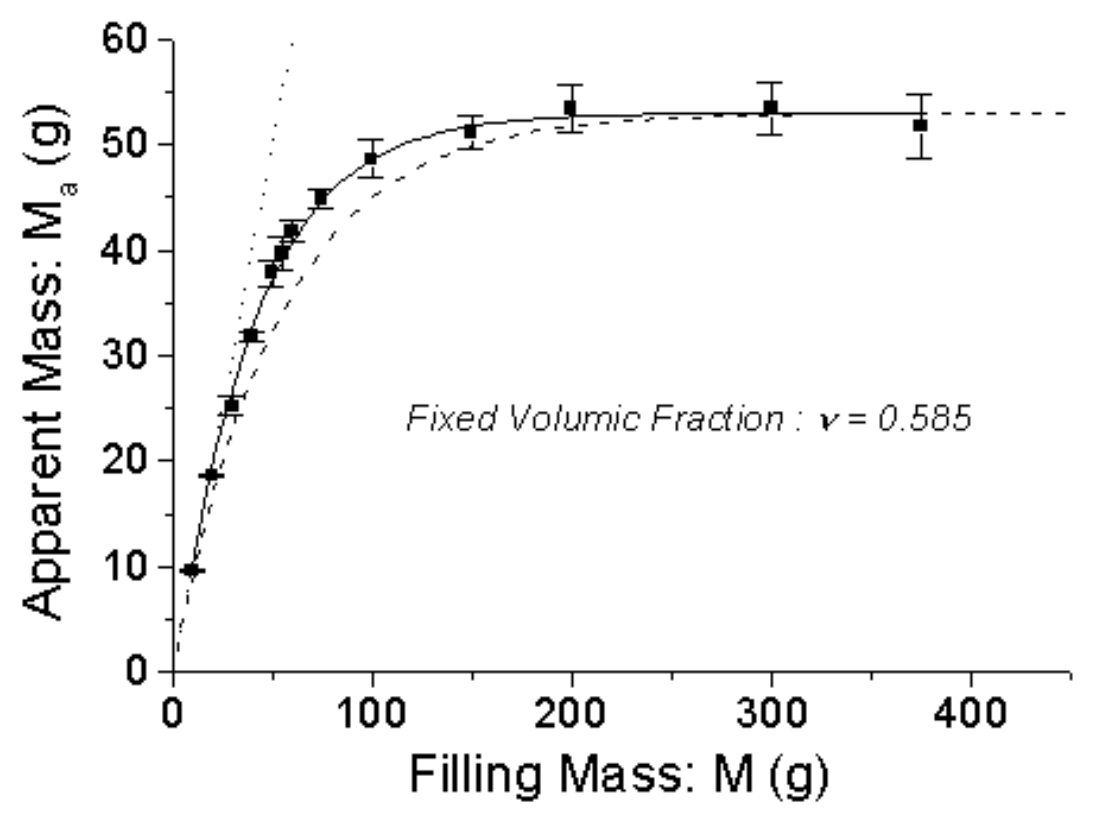

Figure 6: Tapping experiments: Apparent mass $M_{a}$ as a function of the filling mass $M$ for a packing fraction $\nu=0.585 \pm 0.005$. The straight line indicates an hydrostatic behavior; the dashed curve is a fit with Janssen prediction (equation 2) where $M_{\infty}=53 g$; the thin solid curve is a fit with the two-parameter model (equation 3) where $M_{0}=13.4 \mathrm{~g}$ and $M_{\infty}^{C}=39.6 \mathrm{~g}$. 

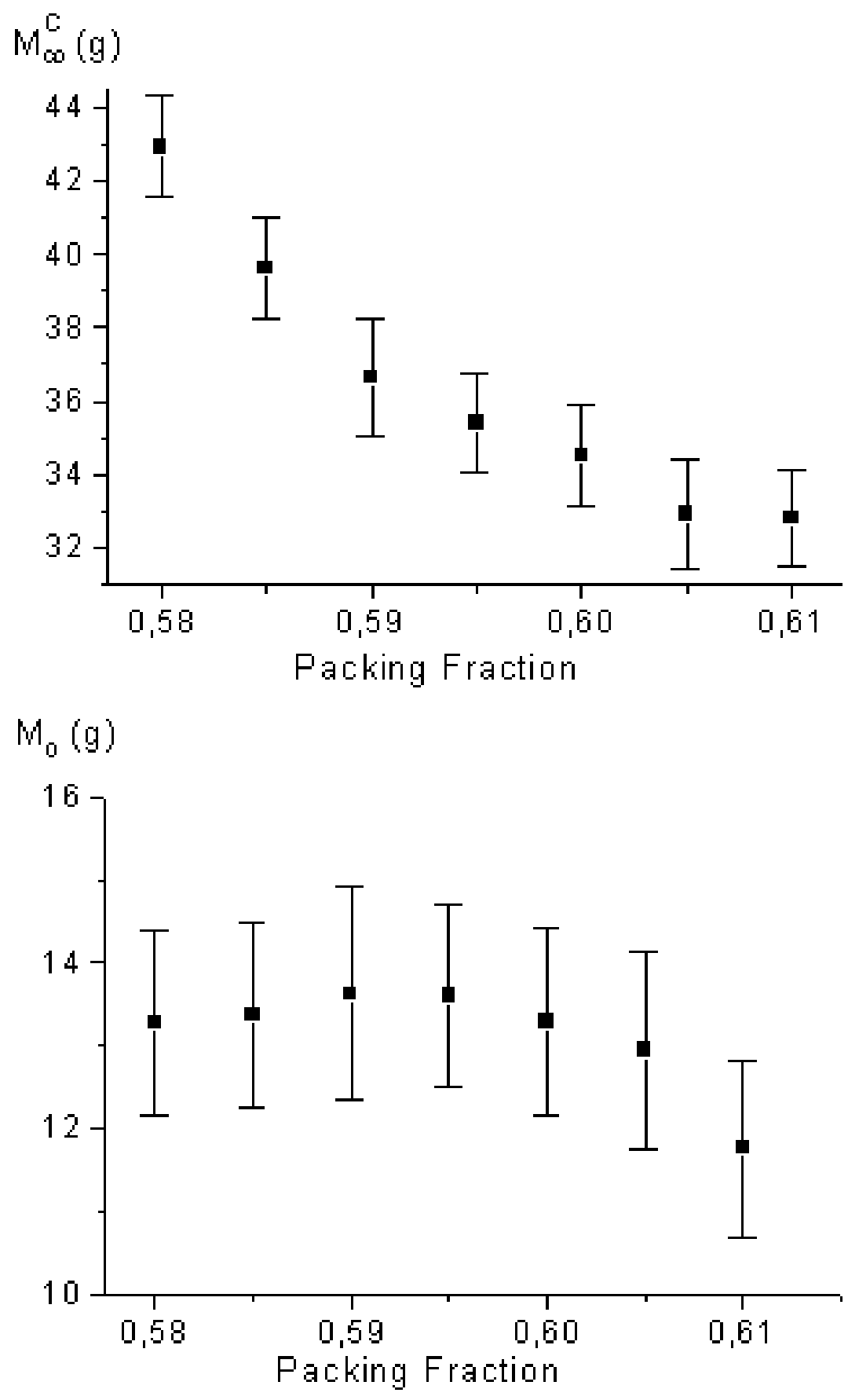

Figure 7: Tapping experiments: Dependency of the two parameters $M_{0}$ and $M_{\infty}^{C}$ with the packing fraction. 


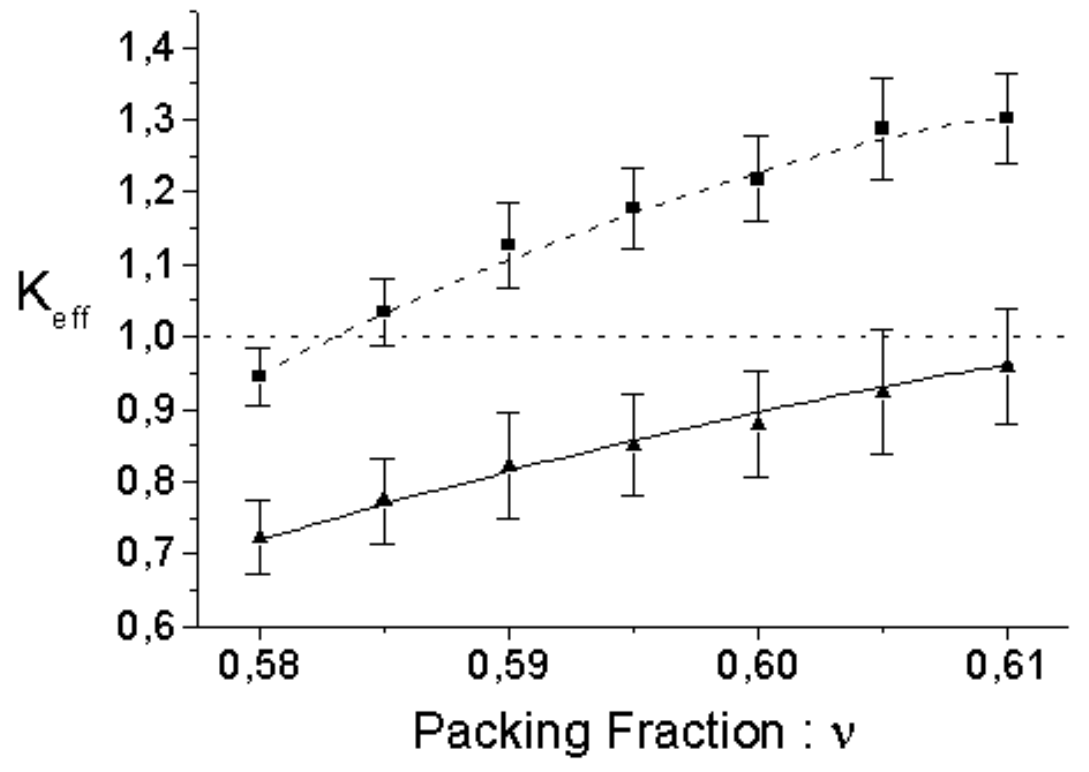

Figure 8: Tapping experiments: The effective Janssen coefficient $K_{\text {eff }}$ as a function of the packing fraction: ( $\mathbf{\Delta}) K_{\text {eff }}$ estimated from the Janssen saturation mass $M_{\infty} ;(\mathbf{\square}) K_{\text {eff }}$ estimated from the corrected saturation mass of the two-parameter model $M_{\infty}^{C}$. 